\title{
Modified Criterion of Hypothesis Testing for Signal Sensing in Cognitive Radio
}

\author{
Mohammed Alamgir, Michael Faulkner, Phillip Conder \\ Center for Telecommunications and Microelectronics \\ Victoria University, Melbourne, Australia \\ Email: mohammed.alamgir@live.vu.edu.au, \\ \{mike.faulkner, phillip.conder\}@vu.edu.au
}

\author{
Peter Smith \\ Department of Electrical and Computer Engineering \\ University of Canterbury \\ Christchurch, New Zealand. \\ Email:peter@elec.canterbury.ac.nz
}

\begin{abstract}
Signal detection problems are traditionally viewed as statistical hypothesis testing. In absence of the a priori probabilities, such as in radar, the Neyman-Pearson criterion is used where a certain false alarm probability is set, and the probability of detection is maximised. In signal sensing problems of cognitive radio, the main constraint is to avoid the interference with the primary user. Once this constraint is met, a cognitive radio can maximise its own chance of finding an empty spectrum. In this paper we emphasise this view of the signal sensing problem and modify the criterion such that a maximum miss-detection rate is specified. We have reformulated the energy detector showing that the sensing results have more meaningful explanations under the modified criterion. The effects of measurement errors are also considered.
\end{abstract}

Index Terms-Cognitive Radio, signal detection, NeymanPearson, hypothesis testing, energy detector.

\section{INTRODUCTION}

In wireless communication the demands for greater speed, more reliability and wider coverage are ever increasing and so is the need for new spectra. But there is very limited amount of unallocated spectrum available in the usable bands of $<$ $3 \mathrm{GHz}$ [1]. At the same time, the utilisation of the allocated spectra is very low as found in [2] and other studies. Cognitive radio (CR) is being thought as a communication paradigm for the future where a spectrum that has been licensed to a primary user can be accessed by a secondary user when idle [3]. The secondary user must not use a spectrum which is already in use, and must free up the spectrum when the primary user begins using it. The regulators, such as the FCC in the US, ACMA in Australia are currently studying the implications of allowing such spectrum sharing [4].

The problem of signal detection has been studied well and applied to radar applications for detecting targets [5][6]. Two types of errors are possible in a simple detection: false alarm and miss-detection. A false alarm occurs when the detector reports the presence of a target while it is absent in reality. A miss-detection is a failure of the detector when a target is present. A false alarm causes an action, for example, in defense, a missile is fired wasting resources. On the other hand, a miss-detection results in loss or causalities. Total avoidance of any loss is always desirable, but the miss-detection probability can not be made zero for a finite detection period. For a given detection period the miss-detection probability can be reduced by allowing increased false alarms, which again costs more resources. Since both of the errors can not be minimised at the same time, a certain false alarm rate is permitted depending on the operational budget, and the probability of miss-detection is minimised.

In CR, a secondary user finds an empty spectrum by sensing the media for possible signals from primary users. A false alarm in such case only means that the secondary user would not transmit in an empty spectrum during an idle period. This would only affect the throughput of the CR, but would not have any impact on the primary user. However, if the secondary user misses a primary signal and starts transmission, then it would cause interference with the primary user. Should future spectrum licenses allow the operation of cognitive radio, we hypothesise that any potential primary users would seek assurances on the maximum level of anticipated interference which the regulators would need to specify in the licensing conditions.

Specifying a false alarm rate helps a secondary user maintain its own throughput; it does not ensure anything about the interference with the primary user. Thus, the constraint of operation of the CR is not met. Recent CR studies seem to miss this point, and continue to report sensing results by specifying false alarm rates [7]. To guarantee that the primary user is not interfered with more than a certain fraction of time, the secondary user needs to have its miss-detection rate specified instead of the false alarm rate. In this paper, we reframe the signal detection problem, and argue that the new view captures the constraints on CR more naturally than the view of the radar target detection problem.

This rest of the document is organised as follows: The detection model and criterion are given in Section II, Section III presents the energy detector under the modified criterion. Effects of measurement errors are given in Section IV, results are shown and discussed in Section V followed by concluding remarks in Section VI.

\section{Detection Model And Decision CRiterion}

Signal detection is usually viewed as a decision theoretic problem, and commonly modelled to have four components: a source that generates outputs (called hypotheses), a probabilistic mechanism, an observation space and a decision rule 


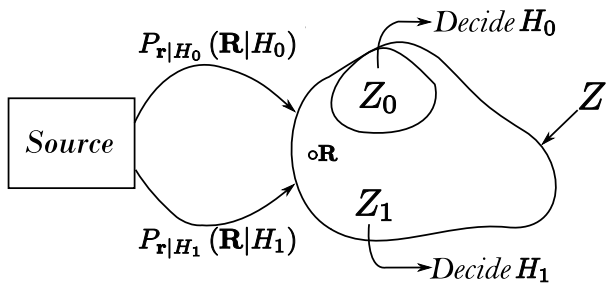

Fig. 1. Decision regions.

(Fig. 1) [8]. The probabilistic mechanism transforms each output of the source into a point in the observation space which is the only thing accessible to a tester.

Let us consider an observation vector $\mathbf{r}$ of $N$ values generated by the probability density functions (PDF) $p_{\mathbf{r} \mid H_{0}}\left(\mathbf{R} \mid H_{0}\right)$ and $p_{\mathbf{r} \mid H_{1}}\left(\mathbf{R} \mid H_{1}\right)$, where $\mathbf{R}$ is a random variable. Here $H_{0}$ and $H_{1}$ are the hypothesis that the primary signal is absent and present, respectively. Based on $\mathbf{r}$, the test must choose either $H_{0}$ or $H_{1}$ corresponding to the decision region $Z_{0}$ and $Z_{1}$. So, for the whole observation space $Z=Z_{0} \cup Z_{1}$. There are four possibilities in making the decision:

1) $H_{0}$ is true; $H_{0}$ is chosen;

2) $H_{0}$ is true; $H_{1}$ is chosen;

3) $H_{1}$ is true; $H_{1}$ is chosen;

4) $H_{1}$ is true; $H_{0}$ is chosen;

where the first and third are correct, the second and fourth are wrong. We call the first case an acquisition since this is the situation where a cognitive radio finds a spectrum empty. The rest of the cases are respectively called false alarm, detection and miss-detection. We have the probability of acquisition,

$$
P_{A}=\int_{Z_{0}} p_{\mathbf{r} \mid H_{0}}\left(\mathbf{R} \mid H_{0}\right) d \mathbf{R},
$$

the probability of false alarm,

$$
P_{F}=\int_{Z_{1}} p_{\mathbf{r} \mid H_{0}}\left(\mathbf{R} \mid H_{0}\right) d \mathbf{R},
$$

the probability of detection,

$$
P_{D}=\int_{Z_{1}} p_{\mathbf{r} \mid H_{1}}\left(\mathbf{R} \mid H_{1}\right) d \mathbf{R},
$$

and the probability of miss-detection,

$$
P_{M}=\int_{Z_{0}} p_{\mathbf{r} \mid H_{1}}\left(\mathbf{R} \mid H_{1}\right) d \mathbf{R}
$$

and the a priori probabilities $P_{0}=\operatorname{Pr}\left(H_{0}\right)$ and $P_{1}=$ $\operatorname{Pr}\left(H_{1}\right)$. Let us assign some cost $C_{i j}$ to choosing $H_{i}$ whereas $H_{j}$ was true. Logically, the cost of wrong decisions are higher than the correct ones, so $C_{10}>C_{00}$ and $C_{01}>C_{11}$. The total cost of making a decision

$$
\begin{aligned}
T C= & C_{00} P_{0} \operatorname{Pr}\left(\text { choose } H_{0} \mid H_{0} \text { is true }\right)+ \\
& C_{10} P_{0} \operatorname{Pr}\left(\text { choose } H_{1} \mid H_{0} \text { is true }\right)+ \\
& C_{11} P_{1} \operatorname{Pr}\left(\text { choose } H_{1} \mid H_{1} \text { is true }\right)+ \\
& C_{01} P_{1} \operatorname{Pr}\left(\text { choose } H_{0} \mid H_{1} \text { is true }\right)
\end{aligned}
$$

or

$$
\begin{array}{r}
T C=C_{00} P_{0} P_{A}+C_{10} P_{0} P_{F}+ \\
C_{11} P_{1} P_{D}+C_{01} P_{1} P_{M}
\end{array}
$$

which after using the fact $P_{A}+P_{F}=1$ and $P_{D}+P_{M}=1$ becomes,

$$
\begin{array}{r}
T C=P_{0} C_{00}+P_{1} C_{11}+P_{1}\left(C_{01}-C_{11}\right) P_{M}+ \\
P_{0}\left(C_{10}-C_{00}\right)\left(1-P_{F}\right) .
\end{array}
$$

In the Neyman-Pearson criterion [8], $P_{F}$ is subjected to a constraint $P_{F}=\alpha^{\prime} \leq \alpha$ for a given $\alpha$, and then $P_{D}$ is maximised. However, it is $P_{M}$ that determines the interference perceived by the primary user. Therefore, the criterion of subjecting $P_{F}$ to a certain value does not meet any interference requirement. To meet the interference obligation, we subject $P_{M}=\alpha^{\prime} \leq \alpha$ and design a test that maximises $P_{A}$ (or equivalently minimises $P_{F}$ ). For this modified criterion, we need to minimise

$$
\begin{aligned}
F= & P_{F}+\lambda^{\prime}\left[P_{M}-\alpha^{\prime}\right] \\
= & \int_{Z_{1}} p_{\mathbf{r} \mid H_{0}}\left(\mathbf{R} \mid H_{0}\right) d \mathbf{R}+ \\
& \lambda^{\prime}\left[\int_{Z_{0}} p_{\mathbf{r} \mid H_{1}}\left(\mathbf{R} \mid H_{1}\right) d \mathbf{R}-\alpha^{\prime}\right] .
\end{aligned}
$$

Obviously, if $P_{M}=\alpha^{\prime}$, then minimising $F$ minimises $P_{F}$. Since, $Z_{0}=Z-Z_{1}$ (where - means the set difference operation), from (8) we have

$$
\begin{aligned}
F & =\int_{Z_{1}} p_{\mathbf{r} \mid H_{0}}\left(\mathbf{R} \mid H_{0}\right) d \mathbf{R}+\lambda^{\prime}\left[\int_{Z-Z_{1}} p_{\mathbf{r} \mid H_{1}}\left(\mathbf{R} \mid H_{1}\right) d \mathbf{R}-\alpha^{\prime}\right] \\
& =\lambda^{\prime}\left(1-\alpha^{\prime}\right)+\int_{Z_{1}}[\underbrace{p_{\mathbf{r} \mid H_{0}}\left(\mathbf{R} \mid H_{0}\right)}_{A}-\underbrace{\lambda^{\prime} p_{\mathbf{r} \mid H_{1}}\left(\mathbf{R} \mid H_{1}\right)}_{B}] d \mathbf{R}
\end{aligned}
$$

To minimise $F$, we assign a point $\mathbf{r}$ to $Z_{1}$ that results in $B>A$. This is because we would like to make the term $A-B$ negative for a positive $\lambda^{\prime}$. Points that make $A>B$ go to $Z_{0}$, and the points for $A=B$ can arbitrarily go to either $Z_{1}$ or $Z_{0}$. Therefore, we choose $H_{1}$ when

$$
p_{\mathbf{r} \mid H_{0}}\left(\mathbf{R} \mid H_{0}\right)-\lambda^{\prime} p_{\mathbf{r} \mid H_{1}}\left(\mathbf{R} \mid H_{1}\right)<0
$$

or,

$$
\frac{p_{\mathbf{r} \mid H_{1}}\left(\mathbf{R} \mid H_{1}\right)}{p_{\mathbf{r} \mid H_{0}}\left(\mathbf{R} \mid H_{0}\right)}>\lambda
$$

where we let $\lambda=\frac{1}{\lambda^{\prime}}$ since it is the sign of $\lambda^{\prime}$ that determines the minimisation. This is the same likelihood ratio that one obtains for the Neyman-Pearson criterion.

The specification of $P_{M}$ instead of $P_{F}$ for meeting the interference obligation can also be explained graphically. Fig. 2 shows the PDFs of $H_{0}$ and $H_{1}$ and the different error regions. The threshold $\lambda$ can be specified from either of the shaded regions representing $P_{F}$ and $P_{M}$. However, for guarantying 


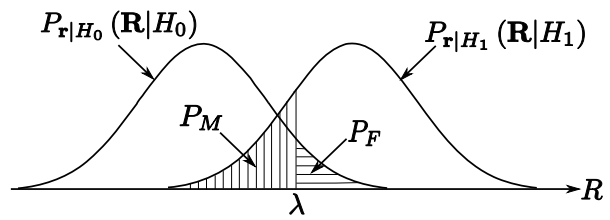

Fig. 2. PDF of $H_{0}$ and $H_{1}$ and the error areas.

a specified maximum tolerable level of interference, we must have the $P_{M}$ specified.

In radar applications, the area $P_{F}$ is usually taken as given because the PDF of $H_{0}$ is commonly known. The other reason is that a radar does not care anything about interfering with the target. For signal sensing problem in CR the primary user can not be ignored, thus the area $P_{M}$ should be specified to limit the interference. This, will require the PDF of $H_{1}$.

\section{ENERGY DETECTOR}

Given $N$ samples $x_{i}$ and the hypotheses

$$
\begin{aligned}
& H_{0}: x_{i}=n_{i} \\
& H_{1}: x_{i}=s_{i}+n_{i}
\end{aligned}
$$

where $n_{i}$ is the noise, $s_{i}$ is the primary signal, and $i=$ $1,2, \cdots, N$. If the samples are Gaussian and independently and identically distributed (IID), the energy detector (ED) chooses $H_{1}$ when

$$
T=\sum_{i=1}^{N} V_{i} \geq \lambda
$$

where we let $V_{i}=x_{i}^{2}$, and $\lambda$ is the decision threshold to be determined.

If the variance of the noise $\sigma_{n}^{2}$, and that of the primary signal $\sigma_{s}^{2}$ are known, the PDF of the decision (13) has $\chi^{2}$ distribution [9]. For reliable detection the sensing time needs to be long, hence the number of samples $N$ is assumed large enough to approximate the PDFs with normal distributions [10].

Under $H_{0}$, the mean, $E\left[V_{i}\right]=\sigma_{n}^{2}$ and the variance, $E\left[\left(V_{i}-\right.\right.$ $\left.\left.E\left[V_{i}\right]\right)^{2}\right]=2 \sigma_{n}^{4}$ where we applied the fact that cumulant of order higher than two is zero for Gaussian variables. Since $V_{i}$ s are assumed IID, the mean and variance of $T$ are $\mu_{0}=$ $N \sigma_{n}^{2}$ and $\sigma_{0}^{2}=N \sigma_{n}^{4}$, respectively. Similarly, under $H_{1}, T$ is normally distributed with mean, $\mu_{1}=N\left(\sigma_{n}^{2}+\sigma_{s}^{2}\right)$ and variance, $\sigma_{1}^{2}=N\left(\sigma_{n}^{2}+\sigma_{s}^{2}\right)^{2}$. Explicitly

$$
\begin{aligned}
& P D F_{H_{0}}(t)=\frac{1}{\sqrt{2 \pi} \sigma_{0}} \exp \left[-\frac{\left(t-\mu_{0}\right)^{2}}{2 \sigma_{0}^{2}}\right] \\
& P D F_{H_{1}}(t)=\frac{1}{\sqrt{2 \pi} \sigma_{1}} \exp \left[-\frac{\left(t-\mu_{1}\right)^{2}}{2 \sigma_{1}^{2}}\right]
\end{aligned}
$$

where $t$ is the real variable for random variable $T$.

The probability of miss-detection, $P_{M}$, and the probability of acquisition, $P_{A}$, are given by

$$
P_{M}=R\left(\frac{\lambda-\mu_{1}}{\sigma_{1}}\right)
$$

and

$$
P_{A}=R\left(\frac{\lambda-\mu_{0}}{\sigma_{0}}\right)
$$

where

$$
R(l)=\frac{1}{\sqrt{2 \pi}} \int_{0}^{l} e^{-y^{2} / 2} d y .
$$

In traditional energy detection, the threshold $\lambda$ is calculated for a specified false alarm rate. Let us call this the Original Criterion (OC). For CR, if we choose $P_{M \max }$ as the maximum miss-detection rate that meets the interference obligation to the primary user, then under this Modified Criterion (MC), from (15) the decision threshold is

$$
\hat{\lambda}=\mu_{1}+\sigma_{1} R^{-1}\left(P_{\text {Mmax }}\right) .
$$

Under this criterion, for setting the proper threshold the PDF of $H_{1}$ is needed which in turn requires the estimation of $\sigma_{n}^{2}+$ $\sigma_{s}^{2}$. With this $\hat{\lambda}$, the acquisition probability can be found from (16).

\section{EfFects of MeAsurement ERror}

Under the original criterion, the decision threshold is proportional to the noise power. If the noise power is overestimated, the resultant false alarm rate is lower than the desired, and so is the probability of detection. In the case of under-estimation, the resultant probability of false alarm and detection, both go high [6]. For a CR using the OC, an overestimation is prohibitive, since it would mean lower probability of detection thus risk of exerting more interference. It can however, underestimate the noise at the expense of higher false alarms, thereby reducing its own throughput.

Similarly, under the modified criterion, an overestimated $\sigma_{s n}^{2}$ would result in $P_{M}>P_{M \max }$ which means more interference than the specified level. If $\sigma_{s n}^{2}$ is underestimated, then $P_{M}<P_{M \max }$ which is good for the primary user; but for the cognitive radio now the probability of acquisition would be low.

Let us consider the measured power, $\sigma^{2}=\epsilon \sigma^{2}$, where the error $\epsilon$ (expressed in $\mathrm{dB}$ ) is a uniform random variable in an interval $[-B,+B]$. For noise, the nominal values of $B$ has been found around 1 to $2 \mathrm{~dB}$ [11]. When measuring combined signal and noise power, we can expect similar error.

\section{Results And Discussion}

We evaluate the criteria by simulating an energy detector. A nominal noise power is set, and the corresponding signal power is calculated for a few signal to noise ratio (SNR) values.

In the first set of experiment we evaluate the effect of measurement uncertainty. Under the OC, the decision thresholds are calculated for a range of $P_{F}$ values using only the noise power. The false alarm events are counted after running the test on only noise. Under the MC, the decision thresholds are calculated using (18) for a range of $P_{M}$ values using the combined signal and noise power. The test is run on combined signal and noise, and the event of miss-detection is counted. 


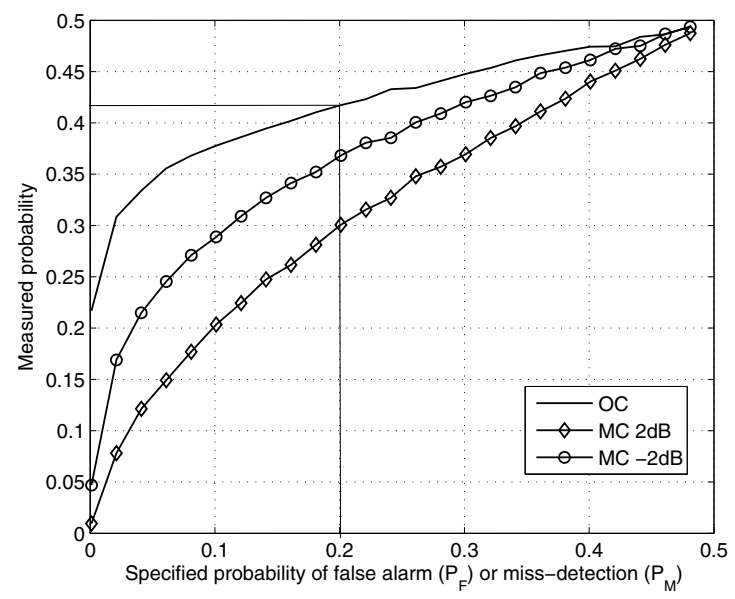

Fig. 3. Measured probability of miss and false alarm. SNR values are $-2,0$ and $2 \mathrm{~dB}$. Uncertainty, $B=1 \mathrm{~dB}$.

Fig. 3 shows how a $1 \mathrm{~dB}$ uncertainty in the noise measurement affects the false alarm (for OC) and miss-detection rates (for MC). For example, a specified false alarm rate of 0.2 leads to an actual observed false alarm rate of about 0.41 . For MC, the miss-detection rate degrades less because the combined power is always greater than the noise power alone.

The $\mathrm{SNR}=2 \mathrm{~dB}$ trace in Fig. 2 implies that the measured miss-detection probabilities are close to the specified values for a strong primary signal. Under such condition, a secondary user using the $\mathrm{MC}$ will be able to guarantee the interference obligation. The OC can not be used in such scenario since no matter how small or large the specified false alarm rate is, it does not guarantee any kind of immunity to the primary user. If the primary signal is weak, then the MC is no worse than the OC.

The MC needs to know the PDF of $H_{1}$, which in present case is the PDF of the energy of the primary signal. This can be learnt in a static environment, but could be difficult in a mobile channel where the average signal power changes. Therefore, MC should be applied to tests where the PDF of $H_{1}$ is readily available. It is also possible to design sensing methods where the PDF of $H_{1}$ is more robust to changes in the signal level [12].

The sensing performance for the $\mathrm{MC}$ can be shown by plotting probability of acquisition against specified miss-detection rates. The average SNR and number of samples can be chosen as the parameter. For example, Fig. 4 shows the acquisition performance of an energy detector under the $\mathrm{MC}$ for different average SNRs. This plot is more appealing than a $P_{D}$ versus $P_{F}$ plot since the relations between the pair of probabilities $\left(P_{D}, P_{A}\right)$ and $\left(P_{F}, P_{M}\right)$ are not always obvious.

\section{CONCLUSION}

We emphasised that it is the miss-detection rate that determines the level of interference a secondary user might have with a primary user. It was hypothesised that the regulators

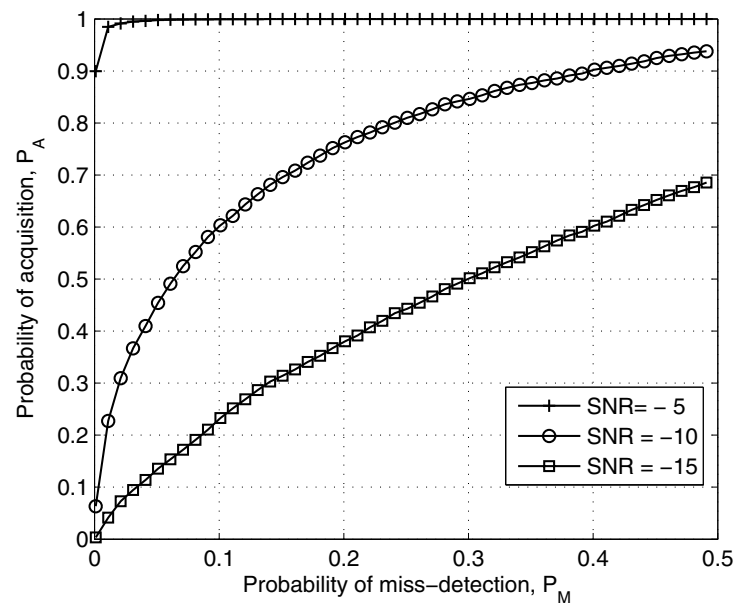

Fig. 4. Sensing performance of the modified criterion. SNR values are -5 , -10 and $-15 \mathrm{~dB}$. Uncertainty, $B=1 \mathrm{~dB}, N=500$.

would specify a maximum miss-detection rate for cognitive radio operators to guarantee the rights of the primary users. With this view, we have proposed a modification to the common signal sensing criterion that better suits the need of cognitive radio. The modified criterion ensures that a secondary user does not exceed a specified maximum interference to the primary users. The energy detector has been reformulated as a demonstration of the modified criterion. The criterion is applicable to any sensing method as long as the PDF of $H_{1}$ is known.

\section{REFERENCES}

[1] FCC, "United States Frequency Allocations- The Radio Spectrum," Available online: http://www.ntia.doc.gov/osmhome/allochrt.pdf

[2] D. Cabric, S. M. Mishra and R. W. Brodersen, "Implementation Issues in Spectrum Sensing for Cognitive Radios," 38th Annual Asilomar Conference on Signals, Systems and Computers, November 2004.

[3] J. Mitola and G.Q. Maguire, "Cognitive radios: making software radios more personal," IEEE Personal Communications, vol. 6, no. 4, pp. 13-18, 1999.

[4] FCC, "Facilitating opportunities for flexible, efficient, and reliable spectrum use employing cognitive radio technologies, notice of proposed rule making and order," in FCC 03-322, December 2003.

[5] W. A. Gardner, "Signal Interception: A Unifying Theoretical Framework for Feature Detection," IEEE Transaction on Communications, vol. 36, no. 8, pp. 897-906, August 1988.

[6] A. Sonnenschein and P. M. Fishman, "Radiometric Detection of SpreadSpectrum Signals in Noise Uncertain Power," IEEE Trans. on Aerospace and Elec. Systems, vol. 28, no. 3, July 1992.

[7] S. Shellhammer, G. Chouinard, M. Muterspaugh, and M. Ghosh, 'Spectrum sensing Simulation model,' available online: http://grouper.ieee.org/groups/802/22/, July 2006.

[8] H. L. Van Trees, Detection, Estimation, and Modulation Theory- Part I. Detection, Estimation, and Linear Modulation Theory, John Wiely, New York, 2001.

[9] S. M. Kay, "Fundamentals of Statistical Signal Processing, Volume 2: Detection Theory," PTR Prentice Hall, Upper Saddle River, New Jersey, 1998.

[10] H. Urkowitz, "Energy Detection of Unknown Deterministic Signals," Proc. of the IEEE, vol. 55, pp. 523-531, April 1967.

[11] IEEE 802.22 Wireless RAN, Functional requirements for the 802.22 WRAN standard, IEEE 802.22- 05/0007r46, October 2005.

[12] M. Alamgir, M. Falkner and P. Conder, 'Signal Sensing for Cognitive Radios Using Synthetic Multipath,' in Proc. of Int. Conf. on Signal Proc. and Comm. Systems, ICSPCS'08, Gold Coast, Australia, December 2008. 